This is the author's final, peer-reviewed manuscript as accepted for publication. The publisher-formatted version may be available through the publisher's web site or your institution's library.

\title{
Client and therapist initial experience of using mindfulness in therapy
}

Kyle Horst, Kimmery Newsom, Sandra Stith

\section{How to cite this manuscript}

If you make reference to this version of the manuscript, use the following information:

Horst, K., Newsom, K., \& Stith, S. (2013). Client and therapist initial experience of using mindfulness in therapy. Retrieved from http://krex.ksu.edu

\section{Published Version Information}

Citation: Horst, K., Newsom, K., \& Stith, S. (2013). Client and therapist initial experience of using mindfulness in therapy. Psychotherapy Research, 23(4), 369-380.

Copyright: (C) 2013 Society for Psychotherapy Research

Digital Object Identifier (DOI): doi:10.1080/10503307.2013.784420

Publisher's Link: http://www.tandfonline.com/doi/full/10.1080/10503307.2013.784420

This item was retrieved from the K-State Research Exchange (K-REx), the institutional repository of Kansas State University. K-REx is available at http://krex.ksu.edu 
Client and Therapist Initial Experience of Using Mindfulness in Therapy

\author{
Kyle Horst \\ Kimmery Newsom \\ Sandra Stith \\ Kansas State University
}




\begin{abstract}
Literature has demonstrated the influence of mindfulness practice on both clients and therapists; however, no literature has considered the experience of mindfulness in therapy from both members of the therapeutic dyad. The present study interviewed five separate therapy dyads $(n=10)$ regarding their experience of first using mindfulness in session. Participants, both clients and therapists, had little-to-no experience with mindfulness prior to the study. Interview transcripts were analyzed and common themes were derived. Results indicated that both clients and therapist felt that the practice of mindfulness had an impact on the therapeutic relationship. . Furthermore, participants also suggested ways in which therapists may better utilize mindfulness in therapy.
\end{abstract}

Keywords: mindfulness, therapy dyad, therapeutic relationship, client experience 


\section{Client and Therapist Experience of Mindfulness}

\section{Introduction}

The increased attention given to eastern philosophy as applied to health and psychology has introduced and popularized the concept of mindfulness. Studies have begun to demonstrate the efficacy of mindfulness-based interventions for a variety of disorders (see Baer, 2003). Mindfulness has also been suggested as a helpful addition to supplement existing treatment models and techniques (Germer, 2005).

Unlike other traditional clinical interventions, however, the application of mindfulness has reached beyond direct client outcomes. As literature regarding mindfulness continues to grow, it is becoming increasingly apparent that mindfulness concepts may enhance clinicians’ effectiveness. Studies have begun to demonstrate that health care professionals who practice mindfulness meditation show an increase in empathy, therapeutic presence, self-care behavior, and other health care provider related skills (Block-Lerner, Adair, Plump, Rhatigan, \&Orsillo, 2007; Christopher\& Maris, 2010; Gehart \& McCollum, 2008; Hick, 2008; McCollum \& Gehart, 2010; Shapiro \& Izett, 2008).

Considering the diversity of applications of mindfulness, this study adds to the current literature by focusing on the in-session experience of mindfulness by both client and therapist. As interest in mindfulness-based interventions grows, the need to understand the experience of these interventions in therapy also grows. The aim of this study is to investigate how both clients and therapists new to using mindfulness respond to the experience of mindfulness exercises. To date, only a few studies have utilized qualitative methodology to investigate mindfulness techniques in psychotherapy. For the present study, we interviewed five client/therapist dyads regarding their experiences of first using mindfulness in session. This exploratory study is one 
of the first to investigate both client and therapist perspective on utilizing mindfulness interventions in session.

\section{Background}

Mindfulness

Mindfulness has been described as "paying attention in a particular way: on purpose, in the present moment, and non-judgmentally” (Kabat-Zinn, 1994, p. 4). Mindfulness applications for mental health have taken a variety of forms and include everything from regular in-session guided meditations to informal discussions of "being-present” in daily life. Most mindfulness interventions, however, include two components: present-being (attention) and non-evaluation (Fromm, 2002).

The first component is "present-being”, or focusing on the "here and now". The contrast to present-being is rigid and fixed attention to a specific thought or activity that allows for other present sensations to be ignored (e.g. rumination) (Wilson, 2008). The emphasis in present-being is attuned attention. Practicing mindfulness requires one to turn his/her attention to the present moment and all the thoughts and sensations that are attributed with "being here”. Germer (2005) explains that most mindfulness exercises include three steps to help with attention: “stop, observe, and return” (p. 116). The first step, “stop”, involves disengaging from any thought or behavior that may serve as a distraction. Typically, this is accomplished by sitting silently or deep breathing - activities that limit distractions and relax the mind and body. The second step is “observe”, or noticing the sensations that accompany that present moment. One may find that while practicing mindfulness his/her mind has wandered out of the "present-being” and to other thoughts; this too is "observed" at which the participant is asked to simply notice this phenomenon and return attention to present-being (Baer, 2005). The last step is the “return”, or 
what is described as the "wake-up". The "return" is the stage between daily living and the first step, the stage where participants understand the need to attune attention back to "present-being". This stage requires a mindfulness of the need to be mindful, and thus the "wake-up is a moment of mindfulness" in and of itself (Germer, 2005, p. 116).

Mindfulness, however, is more than a discipline of finely-tuned attention. Thich Nhat Hanh (2006), a Buddhist monk and mindfulness advocate, writes that "mindfulness means awareness and it also means looking deeply” (p. 9). He points out that mindfulness is not just about "what" is being done (attention/awareness) but "how" it is being done (looking deeply). He later describes "looking deeply" as slowly dissolving the boundaries between the observer and the observed, fully penetrating the object so as to fully know the object (Hanh, 2006). Researchers and clinicians have adopted this principle from a more secular approach and coined it as "non-judgment" or "acceptance". The focus here is on the attitude in which mindfulness is practiced. Baer (2005) states that mindfulness should be approached with "an attitude of friendly, curiosity, interest and acceptance to all observed phenomena, while refraining from evaluation or self-criticism” (p.5). Observed phenomena referred to in this sense often include thoughts and feelings, an important topic when dealing with a clinical population. Often times judgments regarding thoughts and feelings become their own "object” of observation.

In reviewing the mindfulness literature, confusion often arises from an author's failure to delineate mindfulness as a trait, concept, or intervention. At times mindfulness is referred to as an innate personal characteristic (Brown \& Ryan, 2003). Mindfulness has also often referred to as a theoretical construct. Treatment models such as Acceptance and Commitment Therapy (ACT) (Hayes, Strosahl, \& Wilson, 1999) and Mindfulness-Based Cognitive Therapy (MBCT) (Segal, Williams, \& Teasdale, 2002) suggest that increased mindfulness is a mechanism of 
change as well as a theoretical foundation behind specific interventions. Mindfulness is also used to refer to a specific clinical intervention, most often mindfulness-meditation. Much of the empirical literature related to mindfulness has centered on the efficacy of mindfulness skills training in treating specific clinical issues (Baer, 2003). Therapists in the present study utilized mindfulness-based interventions along with other interventions with clients experiencing a variety of clinical issues.

Mindfulness Applications

Mindfulness interventions are currently being studied and applied to a wide variety of clinical disorders and have been successfully applied to those experiencing depression, anxiety, substance abuse, chronic pain, stress, borderline personality disorder, etc. (see Baer, 2003).

One of the first clinical applications of mindfulness was with those suffering from chronic pain. Kabat-Zinn (1982) first developed his Mindfulness-Based Stress Reduction (MBSR) program as a group program that trained participants in mindfulness meditation. MBSR has since evolved to fit a multitude of populations, including cancer patients and women with heart disease (see Baer, 2003). Of the mindfulness-based treatments, MBSR has the most empirical support.

Based on many of the principals developed by MBSR, Mindfulness-Based Cognitive Therapy (MBCT) was created with a specific focus on prevention and relapse of depressive episodes. Segal, Williams, and Teasdale (2002) suggested that mindfulness could allow for increased awareness of depressive symptoms and thus further aid in preventing relapse. Like MBSR, MBCT is a manualized treatment model that utilizes a group setting with a heavy emphasis on homework (Segal, 2002). 
MBSR and MBCT place mindfulness in the center of their conceptual frame, while other models have adopted mindfulness as one of several core tenets. Dialectical Behavioral Therapy (DBT) is one such model that incorporates mindfulness into its practice. DBT was specifically designed for the treatment of Borderline Personality Disorder and utilizes mindfulness as a technique to help clients with acceptance of thoughts as well as a decreasing reactivity. Clients learn to notice their thoughts and feelings before having to act on them (Linehan, 1993). Another treatment model that incorporates mindfulness is Acceptance and Commitment Therapy (ACT) (Hayes, Strosahl, \& Wilson, 1999). ACT focuses on "second order change" or change that is brought on indirectly, and utilizes mindfulness most heavily in its initial phase of "acceptance" where the client learns to release control of the problem (Hayes et al., 1999).

Other researchers have argued that value may lie in mindfulness apart from a specific treatment model and have studied mindfulness as a standalone concept with the potential to be adopted into any clinicians’ existing treatment model (Bishop, Lau, Shapiro, Carlson, Anderson, Carmondy, et al.,2004; Brown \& Ryan, 2003; Ostafin, Chawla, Bowen, Dillworth, Witkiewitz, \& Marlatt, 2006; Shapiro \& Izett, 2008). The current study examines mindfulness as a standalone concept used by therapists working from a variety of theoretical frameworks. Past studies have utilized qualitative methodology to investigate the overall experience of mindfulness-based treatment programs (see Malpass et al., 2012); however, no studies have considered the experience of utilizing mindfulness as a standalone intervention in session. This choice was made not only because of the gap in the literature, but also because we assume that mindfulness techniques are being used by many clinicians outside the framework of a specific mindfulness-based treatment model. Although this assumption is based purely on anecdotal 
evidence, we anticipate the findings to be of value to any clinician or researcher interested in the experience of using mindfulness in psychotherapy.

\section{Mindfulness and the Therapeutic Experience}

As outlined above, the practice of mindfulness entails shifting attention to the present moment and non-evaluative awareness. Hick (2008) explains that it is these qualities that make mindfulness more than a "clinical technique" but instead as a concept that has the potential to impact areas of treatment not traditionally targeted.

Mindfulness interventions may have an impact on the therapeutic relationship when practiced by therapists. Recent studies have begun to demonstrate how mindfulness training can improve therapists’ level of empathy (Block-Lerner, et al., 2007; Shapiro \& Izett, 2008, Lesh 1970), degree of therapeutic presence (McCollum \& Gehart, 2010; Gehart \& McCollum, 2008; Hick, 2008), and help develop overall self-care (Christopher and Maris, 2010; Schure, 2008). Only a few studies have demonstrated that a therapist's personal use of mindfulness is associated with client outcomes (Grepmair,et al., 2007; Ryan, Safron, Doran, and Muran, 2012). Grepmair and colleagues compared treatment outcomes between therapists who personally utilized mindfulness-meditation and those who did not. Results indicated that mindfulness-meditation practiced by therapists was associated with more positive treatment outcomes with those clients who were specifically being treated for somatization, anxiety, anger, obsessiveness, and psychoticism (Grepmair et al., 2007). Similarly Ryan and colleagues looked at how a therapist’s dispositional mindfulness along with self-affiliation and therapeutic alliance were related to client outcomes. Results indicated that therapist's dispositional mindfulness did not directly affect client symptomology but did impact client's interpersonal functioning. Despite these 
results, less is known about the specific mechanisms in which mindfulness aids to the therapeutic experience (Coffey, Hartman, and Fredrickson, 2010).

Several studies have looked specifically at the experience of mindfulness in session. Malpass and colleagues (2012) recently conducted a meta-ethnography of qualitative studies of participant's experience of MBSR and MBCT. The authors found fourteen original studies that met their inclusion criteria. The study’s participants varied, including those with recurrent depression, HIV-positive youth, and patients with a terminal diagnosis with cancer. The authors constructed a narrative of a patient's experience of mindfulness based on 24 different constructs derived from these fourteen qualitative studies. Within this narrative, the authors outline four pairs of therapeutic processes at work in mindfulness practice: dis-identification and "letting go", facing the difficult through inquiry, present-focused and focusing-in, and becoming bigger than experience and looking at things differently. The authors point out patient's difficulties with mindfulness: the inability to let go of expectations and striving for set goals, feeling overwhelmed by the initial exposure to set habits and coping strategies, and a lack of identification in the group (as both MBSR and MBCT are offered in a group format). Conclusions derived from this synthesis highlight the internal and cognitive effects of mindfulness practice.

Although results from this synthesis add to the understanding of the experience of mindfulness in therapy, several limitations are present. First, the studies were restricted to only mindfulness used in the context of MBSR and MBCT programs; however, as stated above, mindfulness is used in a much broader context than these two programs. One potential influential aspect is that these programs are offered in a group format. We wonder if the experience of practicing mindfulness in a group translates to the experience of mindfulness in an 
individual therapeutic context. Also, the studies considered in this synthesis use only the client as a source of data. As mentioned above, mindfulness practice is one in which both client and therapist benefit. Thus, in considering the experience of mindfulness in therapy, it would seem appropriate that both the client's and the therapist's experience are considered. Understanding both client's and therapist's experiences might move beyond an understanding of the individual cognitive impact to a broader relational impact of mindfulness.

No studies have yet to consider the impact of mindfulness practice in therapy from both client and therapist's perspectives. All of the above mentioned studies focus specifically on the impact of mindfulness on the therapeutic relationship when practiced by either the therapist or client alone. The present study will be the first to consider the perceptions of both clients and therapists on mindfulness practice in therapy. This exploratory study investigates the experience of mindfulness practice within the therapeutic dyad.

The research question driving this study is: what is the experience of clients and therapists first using mindfulness in psychotherapy?

\section{Method}

\section{Participants}

The study's sample consisted of ten participants, five clients and five corresponding therapists. All but one of the participating therapists were female, and all of the participating clients were female. Participant therapists ranged in age from 23-44, and client participants ranged from 19-56. Further information regarding treatment length, presenting problem, and specifics about the mindfulness exercises that were used are presented in Table 1.

Participant therapists were recruited from a marriage and family therapy training program in the Midwestern region of the U.S. Participation selection criteria included the following: 
participants are over 18 years of age, therapist participants had used some sort of mindfulness exercise in session with client participants at least twice at the time of the interview, and both client and therapist agreed to be interviewed regarding their experience with mindfulness.

The sample was generated by first recruiting participant therapists; after which, participant therapists were asked to identify a client in their current caseload that fit the above mentioned criteria. Participant therapists then discussed with the selected client participant the option of being included in the study. All therapists in the program were invited to participate in the study.

Participants were not directly compensated for their participation, although therapist participants did receive indirect compensation through building mindfulness skills as part of the study. Upon agreeing to participate in the study, both client and therapist participants were given written consent forms and were debriefed regarding the data collection process.

\section{Study Design}

Data for this study were collected via qualitative interviews involving both members of the participating therapy dyad, therapist and client. A discussion among researchers considered the costs and benefits of interviewing each member of the therapeutic dyad together or separately; however, since the focus and novelty of this study includes collecting data from both therapist and client, we felt that interviewing the individuals separately might discount the relational aspects of the experience of mindfulness in therapy. Thus, interviews were conducted with both client and therapist together. Interviews were semi-structured following a guide including specific questions related to the experience of mindfulness in session. Interview questions included, "What was the experience of using mindfulness in session?", "When your therapist first mentioned using mindfulness in session, what were your first reactions?", "How 
did the mindfulness experience meet your expectations?”, “What, in your opinion, contributed to the success (or failure) of using mindfulness in session?", and "what advice would you give to a therapist using mindfulness for the first time?” These questions were used in all the interviews to facilitate conversations about the topics, however, other questions were asked as seemed fit by the interviewer. All interviews were audio-recorded and notes were taken throughout the interview by the interviewer. Interviews took place in the same therapy room at the clinic in which the client was being seen.

Prior to the study, the first author offered a seminar on mindfulness techniques to therapists-in-training, some of which volunteered to be in this study. The seminar was an eightweek group practicum for clinicians interested in learning basic mindfulness skills for their own practice as well as a clinical intervention. This seminar included both didactic learning as well as experiential components. Topics included learning mindfulness concepts (e.g., awareness and acceptance) and experiencing mindfulness exercises (e.g., body-scan and loving-kindness meditations). Students were encouraged to begin developing their own mindfulness practice prior to using it in session. Each week students learned a new concept or application related to mindfulness, experienced a mindfulness exercise, and discussed their own practice. For three of the five participating therapists, this was their first exposure to mindfulness practice. The other two therapist participants had learned mindfulness on their own from books or other resources; however, all therapist participants noted that their utilization of mindfulness exercises in therapy was new..

\section{Data Analysis}

After the interviews were conducted, all recorded audio was transcribed verbatim. Transcripts were then analyzed by the authors utilizing Thematic Analysis (Braun \& Clark, 
2006). All of the authors are licensed Marriage and Family Therapist with varied experience using mindfulness either on their own or in therapy. The authors followed the six phases of thematic analysis suggested by Braun \& Clark (2006): 1) became familiarized with data, 2) generated initial codes, 3) searched for themes, 4) reviewed themes, 5) defined and named themes, and 6) produced the report. The first step involved all three authors reading all the transcripts to become broadly familiar with the data. After doing so, two authors re-read the data and coded anything related to the research question. At this point, the researchers met to discuss potential themes by comparing and contrasting each other's coded segments. Themes were then reviewed by the researchers re-addressing the data, this time looking for evidence of the specific themes. Throughout this process, theme names/definitions were redefined per new discoveries in the data. Researchers then discussed one last time overarching organization of themes into two main categories (discussed below). Finally, the first two researchers summarized the findings into a report, again comparing findings.

\section{Findings}

Analyses revealed several important themes related to client and therapist experience of mindfulness in session. Themes were organized into two overarching categories; "The experience of using mindfulness activities" and "therapist and client suggestions about how mindfulness can be successfully utilized in session". The first theme relates directly to our primary inquiry into how clients and therapists experience mindfulness in session. The second theme was one that emerged during our analysis and indirectly relates to our research question. In general, all participants had positive comments regarding the use of mindfulness. Although our analysis highlights similarities in participant comments, differences in each dyad's length of treatment, presenting problem, and particular use of mindfulness are list in Table 1. 


\section{Experience of Using Mindfulness in Session}

When asked about their experience of using mindfulness in session, participant clients and therapists mentioned several notable themes. Both clients and therapists were asked to reflect on the questions "What was the experience of using mindfulness in session?", "When your therapist first mentioned using mindfulness in session, what were your reactions?", and "How did the mindfulness experience meet your expectations?” Themes derived from these questions include: "Helpful with presenting problem”, “Aided with in-session transitions”, “Facilitated conversation", "Lack of confidence in ability to use or facilitate activities", "calming”, and "slowed session pace".

Helpful with presenting problem

Clients and therapists were asked to discuss their experience with mindfulness and whether their response to the use of it in session was positive or negative for them. As mentioned above, all participants reported that they had a positive experience with mindfulness in session. For therapists, this was expected since they self-selected to participate in the study. Clients' reactions, however, were less predictable yet, their experiences seemed to be congruent with therapists' experiences..

One client discussed how positive it was to use mindfulness in response to managing pain: "Um yeah, I have neuropathy and so that was interesting. I think it was helpful with the pain, I mean it was definitely something different.” One client whose therapist had previously asked her to focus on breathing was asked about the experience of focusing on the body or doing the "body scan”. She offered this insight: “...I liked that better. Just breathing was hard for me cause my mind would go crazy. But when I focused on my body it was easier.” Another client 
was asked whether she had any previous experience with mindfulness and how she felt about the experience. The client responded:

Um, no. I never, this is my first experience with it and I, I really appreciate it. I tend to get, well, I came here convinced I had ADHD (laughing). I tried to convince you [talking to therapist]. But that is just how my mind races. I've let it, my emotions run away with me and that's where all the, so this is good. I'm looking forward to this.

Whether specifically related to their presenting problem or simply as an aid to treatment, participants found the experience of using mindfulness in session positive. Therapist participants mostly agreed with their client's statements regarding how mindfulness related to presenting problems; however, therapists did not always utilize mindfulness in session in hopes of directly impacting the presenting problem. One therapist explains how she simply used mindfulness as a way to "transition into therapy." Helpful with Transitions

Participants noted that using mindfulness in session was "helpful with transitions" in therapy. This was a theme that was common among therapists and clients. One therapist/client dyad discussed how using a mindfulness meditation at the beginning of the session helped them transition from the stresses of life to being present in therapy in this way:

Therapist: I think it just helped us re-focus. I also think the pacing of the session changed a little. We could really slow down too. Like at first it was like a machine-gun of stuff, "this stuff happened, and then this, and this" but then we both breathed and took a moment to reflect.

Client: Yeah, that helped a lot. I come here... and sometimes I'm all over the place. So much is going on with my family too that I usually have a lot to get through here.

These participants viewed the use of mindfulness in session as a means to focus in session.

Clients noted mindfulness exercises eased that transition from whatever was happening prior

(e.g. class, work, commuting, etc.) to session. Participant therapists noted that using mindfulness exercises helped them to prioritize in session. After commenting on how mindfulness helped the 
transition, one therapist participant noted, "Well like, she might have felt more pressure to talk about the "crisis" instead of slowing down and talking about other things that were more important.”

Beyond transition into and out of session, participants also mentioned using mindfulness to ease transitions during session. Specifically, one participant therapeutic dyad mentioned using a mindfulness exercise to transition from talking into an experiential exercise. Both client and therapist noted how the two exercises "go together" and "dove-tailed one another." The therapist participant went on to explain how mindfulness helped with this transition in that she wanted her client "to just start experiencing emotions instead of thinking about them so much.” Both client and therapists recognized the value of the mindfulness exercises in transitioning into therapy and within different activities in session. Therapist participants seem to appreciate the value of this more overtly than client participants.

Facilitated Conversations

Similarly, the use of mindfulness in session was found to impact the session in that it facilitated conversations. Participants reported that conversations about mindfulness concepts were helpful to the overall treatment. One therapist participant commented, "The other thing was that I really felt like the conversation about mindfulness...I mean like talking about what it was and how to do it...that was helpful.” A client participant also noted how mindfulness exercises changed the content of subsequent conversations, “....and even when I have something to talk about, after the mindfulness it might not seem as big of a deal...like it helps clear the way a little.” This client's therapist also noted how mindfulness impacted the subsequent session, "like a lot of times we would do an exercise and she would want to talk about it...and then the whole session was over. So that was nice, like it really facilitated the conversation.” 
It is interesting to note that both clients and therapists appreciated this experience of mindfulness. Both clients' and therapists' comments highlight how the activity of mindfulness was shared with one another. Unlike other therapeutic techniques, this shared experience seemed to be what facilitated conversation in many of the participant dyads. Lack of Confidence in Ability to Use or Facilitate Activities

Another theme that emerged was related to the comfort level of the participants using mindfulness in session for the first time. When asked directly about their experience, therapists were able to talk about the reservations they had initially and any anticipated reactions from the client. One therapist spoke of his experience as follows:

So I had some experience already, but I think I'm still nervous that clients are not going to be open to it. They will be like, "what's this crazy guy talking about?" Part of it is that you are trying to build a relationship you know trust, and so you worry about whether or not they think you are competent. I mean, for clients with experience, it's not as big of a deal. But you take someone... who has never meditated or done anything like it in their life, it might be a big shock. And that's ok it just might be a hurdle.

Clients also felt a degree of self-consciousness using mindfulness for the first time. One client/therapist dyad shared similar doubts about correctly using mindfulness; “Therapist: I think...Am I going to do this right? Is this going to work?” (laughter) Client: Same thing I thought...(laughter).” One therapist participant spoke of the nervousness experienced, coupled with the feeling of anticipation to the exercise:

Yeah, I used a CD. I was nervous because I wanted \{client\} to like it and use it again. So yeah I used a CD to guide the first time and then I led it after that. I would say I really believed in it because I like to do yoga and meditate and think about coming down from the day, so I was looking forward to doing it too.

Similarly, this therapist's client confirmed these feelings:

Yeah, I'm glad we did it with a CD the first time. Cause you just feel awkward at first. I mean it's just, it's just awkward the first time. I mean when you are in the room with someone you don't know well, you usually talk to dissipate the nervous, you are just nervous. You don't very often sit with someone you don't know very well. 
It is important to note that all the participants talked about nervousness with initially using mindfulness, however, most also noted becoming increasingly comfortable with continued use of the exercises. Considering the therapist's and client's relative inexperience with mindfulness, this theme seems appropriate; however, it is interesting to note that many of the therapy dyads mentioned sharing their apprehension with one another, which in turn, seemed to facilitate a more authentic conversation. For several therapists, this vulnerability of disclosing their lack of confidence using mindfulness may have in turn helped develop the therapeutic relationship.

Calming

All participants noted that using mindfulness activities had a calming effect on the session. One client participant recalled that the sessions that included mindfulness exercises were calmer and more thoughtful:

Well, yeah I think they were slower, well, not slower. I guess maybe more calm, more thoughtful. I mean most of the time we did mindfulness at the end so I couldn't really tell you. But the times we did at the beginning or middle I noticed that. But that is why I would ask you to do it. I knew I wouldn't be able to concentrate. I needed that time to refocus, or just let go whatever else was happening.

Another client participant said this regarding the sessions where mindfulness was used as opposed to those where mindfulness was not used, “....it was more relaxed, more focused. I think they were a little slower too. Like after the exercise we both were a little more calm. They were probably easier for her [laughing]. Like I'm sure I was easier to follow after that.” One client noted mindfulness exercises made her sleepy, but also said this regarding the relaxation, “...but really relaxed and like (laughs) I don’t know if that’s like if I was still working at [company name] and I was tired or what but I know that they are really calming and relaxing." 
Therapist participants also noted the calming effect of mindfulness in session, both on themselves and the client. These results seemed consistent regardless of the specific type of mindfulness exercise used or whether it was done at the beginning, middle, or end of session. Client participants, however, had much more to say regarding the relaxation and calming effects than therapist participants.

Slowed Session Pace

Related to the calming effects of mindfulness, participants also noted the pace of session was slower after a mindfulness exercise. One therapist commented regarding this change in pace:

Well, I think it was good. I liked the fact that we started the session off slower. A little more reflection. Almost like, ok, we're here now, everything can calm down. I mean it even helped me too, I have a few sessions before this and it helped come down from those. They are mostly with kids so it's totally different. But I appreciated having a moment to sit and breathe.

One therapist participant also noted how mindfulness improved her ability to listen to her client:

From my end I felt like, that it made me listen to [client] really, really intently. You know, it's like...that experience or that emotion...it's the truth for now. And kind of accepting that and holding that. For me as a therapist, it is really powerful, important, valuable.

Despite not commenting on the calming effects of mindfulness, therapist participants noted the slower session pace much more frequently than did client participants. This is probably because therapists are more aware of the time-management in session as they are more responsible for the course of the session. Beyond this, the sense that a session was "slower" might also indicate a greater sense of emotional depth in session. A slower pace in session might bring with it a more intentionality in the therapeutic conversation.

\section{Utilizing Mindfulness Successfully in Session}

In addition to speaking about their experience using mindfulness exercises in session, client and therapist participants also offered suggestions regarding how to best utilize 
mindfulness in session. Analyses of these themes revealed to us what participants thought as important to the experience of mindfulness in session. Additionally, we felt that including these themes would add to the understanding of how mindfulness is best used in session. Two main interview questions addressed this category of themes: "What, in your opinion, contributed to the success (or failure) of using mindfulness in session?" and "what advice would you give to a therapist using mindfulness for the first time?” Although answers to these questions informed this category of themes, analysis was not limited to only these questions.

Five key themes were identified within this category. Participants noted that part of what contributed to the success of using mindfulness in session was that both clients and therapists were participating in a "shared experience", "the practice was continued" both in and out of session, and the therapist was "flexible" with how mindfulness was utilized. Participants also suggested that therapists using mindfulness for the first time should "preface the experience with an explanation and process the experience" after it is practiced. Lastly, several participant dyads noted the important role "trust" played in practicing mindfulness in session.

Mindfulness as a Shared Experience

Both client and therapist participants commented on the unique experience of practicing an exercise in session together. Clients noted that knowing their therapist was also practicing with them made the exercises more comfortable. One client stated, "I think like what you said, we do it together. That helped. I didn’t think about it much until just now, but that helped. Cause I'm an anxious person, I liked knowing it wasn't just me and that you were there doing it.” Similarly a therapist participant mentioned enjoying the experience of practicing mindfulness together:

I mean I like that it's different. It's nice for me to not have to talk either...I mean not talk like therapy talk, I guess. I still talk 'cause I lead the exercise, but it's different. Plus I get 
to do it too. That's nice for me. It is something we do together, not just something she does and I sit and stare at her.

Much like this participant noted, others also mentioned how mindfulness exercises were "different" in that they involved both client and therapist.

Clients and therapist also shared in their experience of creating a practice of mindfulness outside of session. Several participants noted that conversations about using mindfulness out of session were similar for both clients and therapist. One dyad noted how both individuals had similar struggles maintaining a regular practice of mindfulness. For example, one dyad had similar thoughts of "doing it right" and questions about whether or not practicing mindfulness would "work" - common considerations for first-time mindfulness practitioners (Kabat-Zinn, 1994). Thus even in their doubts and apprehensions about practicing mindfulness, clients and therapist shared in their experience.

Continued Practice

Participants also noted that practicing mindfulness regularly also contributed to its success. Both clients and therapist noted that developing a practice of mindfulness, both in and out of session, was ideal. One dyad highlighted this point by noting:

Client: So I mean, back to the question, I like doing it here too. I mean it is good to do it on my own too. But for a lot of people they might need to practice before doing it on their own.

Therapist: Yeah, I agree. I mean the goal is obviously to have them do it on their own. But you can't expect to do it once and then just become like a mindfulness guru. You need practice. You know I usually start with like a 2 or 3 minute breathing exercise. That alone is a lot for some people. It may take a month or two before moving beyond that.

Additionally, clients and therapists both noted that a therapist's own comfort and experience with mindfulness would translate into a better experience in session. One therapist participant noted this and commented, “...I think that it helps you talk about ... I think there is more like authenticity or something built around talking about your own experience with it 
and...maybe....it would help you understand somebody else's experience with it better if you've had your own or are kind of attuned to the way it impacts you in your life.” One client participant reflected on her therapist's congruence with mindfulness practice and stated, "If I'd known anything about it, I would have known you practiced it yourself.” Fundamentally, participants noted that a therapist's personal use of mindfulness helps "relay it on to \{client\}." Flexibility

Participants also mentioned that therapists should be flexible when using mindfulness in session. For example, one therapist participant recommended not becoming too attached to mindfulness techniques and to be ok "moving on" from the exercise if the client did not find it helpful. This, however, could also be related to the therapist's inexperience with mindfulness. Therapist more experienced with mindfulness might recognize ways in which client discomfort can inform treatment. Clients also mentioned enjoying when the therapist tailored mindfulness exercises to fit the specific needs of treatment. This refers not only to the content of the exercise but also how it was introduced and used in session. For example one client participant spoke of how she appreciated her therapist not prescribing mindfulness as a "homework assignment" but rather a practiced discipline. She stated, "motivation has to be intrinsic, it’s not something that...she never made me feel like that....and I probably wouldn't, if I felt like it was one more assignment I had to do, or a job. Yes, I like the way you put that, self-care. I can do that.” Prefacing/Processing

Part of what made mindfulness a successful endeavor for both client and therapist participant were the conversations before and after the experience. These conversations ranged from preparing the client for mindfulness as well as processing the exercise after. Participants 
noted that these conversations were critical to the successful integration of mindfulness in session.

Several client participants noted how their therapist initially introduced mindfulness as an important element of its success. Clients appreciated hearing a "rationale" for including mindfulness in session, although several noted some "hesitance". For one client, it was helpful that her therapist presented the mindfulness exercise without expectations. She commented, “You don’t want to make someone feel like they aren’t doing this right. You don't want to set up an expectation. Like I think what [therapist] did was explain this as a way we are transitioning into the sand tray. So she explained it and it didn't seem so weird.”

Beyond explaining mindfulness, participants also noted the importance of asking for client's permission. Several client participants appreciated their therapist asking them if they were comfortable with certain aspects of the exercise like dimming the lights or closing their eyes. One client participant in particular highlighted this point when she stated:

I think the first time you said "I can leave the room if you want me to." So she gave me that option, which is nice. But I said 'no', and I can let her know 'it's ok'. But I do think it takes two-way communication, like do we need to dim the lights? What's going to make this more relaxing?

Similarly, participants also noted that asking the client if they would like to continue to practice mindfulness in session was helpful. One participant stated, "ask if they want to do it again. [therapist] asked me after the first time if I wanted to try again another session. That was nice to have my input."

Conversation held after a mindfulness exercise was also perceived as beneficial. Both client and therapist participants appreciated processing mindfulness in session. As noted above, several therapist participants found that the post-mindfulness conversation lead into other relevant discussions for treatment. One therapist participant mentioned, "I think it was 
interesting to talk about it like this or kind of reflect on it because I think that sometimes I'm not aware of the growth that happens umm...until afterwards or until you look back or something...” This particular therapist went on to describe how talking about mindfulness unveiled some of what the client was gleaning from the exercise, which in turn informed future exercises. Trust

Another important element mentioned in the successful utilization of mindfulness in session was the presence of trust between client and therapist. Both client and therapist participants mentioned that practicing mindfulness exercises together in session involves a certain degree of vulnerability. One therapist participant explained this when he said, "Yeah, especially because it was so early, you don't know me and I'm asking to turn the lights off? That could be weird. Close your eyes please. I mean, it is sort of a... it takes some trust.”

Several participants suggested that a certain degree of therapeutic relationship has to be built before using mindfulness exercises in session. A few participants recommended not using mindfulness exercises in the first few sessions with a new client, but waiting to establish a therapeutic relationship. One therapist participant explained,

I think the relationship has to be there...like a pretty strong trusting relationship where you can both be vulnerable. And I think you have to know your client well. Because I knew how to present to [client] and I knew how to say things that would be helpful, like the meadow image I knew would really hit home with [client]. I say the trust has to be there...

This therapist's client followed this statement by saying, "I think that is important, what you said about knowing the person and knowing what works for the person. I think that is what doesn't work sometime when it's just a CD, you know, cause they don't know my body or this or that.” One client noted that with more trust in the therapeutic relationship, she felt comfortable telling her therapist if she didn't want to continue using mindfulness exercises. In elaborating about the 
issue of trust, she stated “Yeah if I felt really anxious, or...I don’t feel like she forced me...or if I had something that was really pressing, we didn't have to do it if I had other things I wanted to talk about.”

\section{Discussion}

Numerous studies have suggested that mindfulness can have a positive impact on a variety of mental health related issues (see Baer, 2003). Additionally, several studies have begun to suggest how mindfulness might influence other elements of therapy not directly related to treatment outcomes (e.g. therapeutic relationship) (Bien, 2006; Fulton, 2005; Gehart and McCollum, 2008). Scholars have considered how the experience of mindfulness in session might reveal more regarding its mechanisms of action (Malpass, et. al., 2012). In this study, we were interested in discovering the experience of therapists and clients new to the experience of using mindfulness in session from both client's and therapist's perspective. Thus, this inquiry offers a more holistic insight to how mindfulness may influences psychotherapy treatment. The findings also suggest several considerations for therapists using mindfulness exercises for the first time.

Unique to the present findings is the indication that the experience of mindfulness in therapy is one shared by clients and therapists. Much of the literature regarding the experience of mindfulness focuses on the individual's internal experience; however, these results suggest that mindfulness may influence session through an impact on the therapeutic relationship. Both client and therapist participant's recognized the influence of one another on their initial experiences of using mindfulness in session.

Participants in this study consistently indicated that the use of mindfulness in session did indeed have an impact on the experience of therapy. In general, study participants found the 
experience of using mindfulness in session positive. Even though they found the experience positive, there was also some apprehension and nervousness experienced among the client/therapist dyads using mindfulness for the first time. For several participants, using mindfulness in session was their first experience with meditation or any other contemplative practice, thus some apprehension is expected. Additionally, some therapists expressed apprehension related to how the client would react or if mindfulness exercises would be effective. This finding is expected considering that participant therapists in our study were therapists-in-training and had all recently learned mindfulness techniques.

Others have suggested that a therapist's own sense of mindfulness might influence sessions (Bruce, Manber, Shapiro, \& Costantino, 2010; Cigolla \& Brown, 2011; Fulton, 2005). Bien (2008) suggested that as therapists adopt a mindful presence, they will also convey greater warmth and empathy toward their client. Cigolla \& Brown (2011) suggest that a therapist's use of mindfulness effects their "way of being" with clients. Similarly, the present findings suggest that practicing mindfulness in session with clients may also effect session in ways beyond treatment outcomes. Many of the clients and therapists agreed that practicing mindfulness in the session provided a sense of calm, facilitated conversation, slowed the pace of the session, and was helpful with transitions.

\section{Limitations and Suggestions for Future Research}

As mentioned earlier, the aim of this study was exploratory in nature. It is the first to consider both clients' and therapists' joint experiences of mindfulness in session. Goals of the study were to enhance our knowledge of how mindfulness might influence therapy. Considering these goals, several limitations are derived from the study. The sample was small (only five therapist-client dyads). All therapist participants were still in training and relatively new to using 
mindfulness in therapy. Thus, results are reflective only of the experience of new therapists using mindfulness for the first time. Additionally, all of the therapists learned to use mindfulness in therapy from the same instructor as part of this research study. Only one therapist participant had prior experience with mindfulness. Thus, some of the results may be in part due to the instructor's methods of teaching mindfulness rather than the use mindfulness alone. Therapist participants self-selected which clients to use mindfulness in session with, thus, some bias as to the openness of the client to mindfulness might also be present. Likewise, therapist participants self-selected to be in the study and were more likely to be open to concepts like mindfulness. Information regarding the models of treatment used by each dyad was not collected, which may have influenced the experience of mindfulness in session. This information was purposefully excluded as we felt it may detract from the main aim of the study. However, we do feel the question of how mindfulness is experienced within the framework of non-mindfulness-based treatment models warrants its own investigation.

Allowing for both the client and therapist participants to be interviewed together presents both strengths and limitations. As mentioned before, this cooperative interview enhances our understanding of the relational aspects of using mindfulness, allowing the dyad to process the experience together as it was experienced together initially. Also, few studies have considered both therapists' and clients' perspectives on using this technique in session, and no study has gathered data simultaneously from client and therapist. Although some of the novelty of the study is maintained in this study design, some limitations may be assumed. One limitation is that, due to the natural difference of power in a therapeutic dyad, some client participants may not have been critical of mindfulness so as not to offend or upset their therapist. However, interviewers were conscious of this differential of power and used different techniques to curb 
this bias, such as asking clients to answer before the therapist, asking the therapist participants to reaffirm with their clients they would not be upset, and gauging the general uneasiness of the client throughout the process. All interviewers reported that both client and therapist participants seemed genuine in their responses. Furthermore, there were no client participants who enjoyed every aspect of the mindfulness exercise fully, indicating that the client participants felt free to criticize the technique on some level. Despite this however, the potential for bias created from interviewing client and therapist together was present.

The present study used both clients and therapists as sources of data while studying mindfulness in therapy. Future research on this topic should continue to consider the relational implications of mindfulness, both in and out of therapy.

\section{Clinical Implications}

Findings of the present study have direct implications on the practice of mindfulness in therapy. We suggest that clinicians considering using mindfulness in their practice review what our participants had to say about the success of mindfulness in session. Participants suggested several considerations for clinicians: conversations about mindfulness (both before and after), maintaining flexibility about using mindfulness, practicing mindfulness on their own, and building trust prior to using mindfulness in session.

Participants recommended that therapists spend time prefacing and processing mindfulness exercises. Several clients expressed the desire to be informed by the therapist regarding the purpose of mindfulness. A few even said that they would like to be asked if they were willing to participate in the process again. This provides the client with a voice and an opinion in the matter related to his or her treatment. It allows the client to inform the therapist and creates a collaborative process out of mindfulness. Similarly, it is important that the therapist 
remain flexible with the use of mindfulness in session. Prescribing a specific "right way" of doing mindfulness is contradictory to the spirit and aim of mindfulness techniques. Furthermore, clients who are struggling with the exercises may feel less inclined to voice these concerns if the therapist is dogmatic with their application of mindfulness in session.

Both client and therapist participants noted the importance of the therapist practicing mindfulness on his or her own. This seems congruent with others who suggest that a therapist's own mindfulness practice might positively influence session (Bruce, et al. 2010; Crane \& Elias, 2006; Shapiro \& Izett, 2008). An issue of authenticity seems implied in the findings; those therapists who espouse mindfulness should do so genuinely. This might become difficult for therapists who have not practiced on their own.

Another derived implication for clinicians is the consideration of trust. Others have indicated that mindfulness practice can influence the therapeutic relationship (Bien, 2006; Fulton, 2005; Gehart \& McCollum, 2008; Surrey 2005). Our findings suggest a somewhat inverse relationship; our participants suggested that an established therapeutic relationship was important for successfully using mindfulness in session. Considering both past findings and these results, the relationship between mindfulness and the therapeutic relationship may be seen as bi-directional in that both variables influence one another. 


\section{References}

Baer, R. A. (2003). Mindfulness training as a clinical intervention: A conceptual and empirical review. Clinical Psychology: Science and Practice, 10, 125-143.

Baer, R.A. (2005). Mindfulness-based Treatment Approaches: a clinicians guide to evidence base and applications. Burlington, MA: Elsevier.

Bien, T. (2006). Mindful Therapy: A guide for therapists and helping professionals. Somerville, MA: Wisdom.

Bien, T. (2008). The four immeasurable minds: Preparing to be present in psychotherapy. In S.F. Hick \& T. Bien (Eds.), Mindfulness and the therapeutic relationship (pp. 37-54). London: The Guilford Press.

Bishop, S. R., Lau, M. Shapiro, S., Carlson, L., Anderson, N. D., Carmondy, J., et al. (2004). Mindfulness: A proposed operational definition. Clinical Psychology: Science and Practice, 11, 230-241.

Block-Lerner, J., Adair, C., Plump, J.C., Rhatigan, D.L., \& Orsillo, S.M. (2007). The case for mindfulness-based approaches in the cultivation of empathy: Does nonjudgmental, present moment-to-moment awareness increase capacity for perspective-taking and empathic concern? Journal of Marital and Family Therapy, 33(4), 501-516.

Bruce, N.G., Manber, R., Shapiro, S.L., \& Costantino, M.J. (2010). Psychotherapists mindfulness and the psychotherapy process. Psychotherapy: Theory, Research, Practice, Training, 47, 83-97.

Braun, V., \& Clark, V. (2006). Using thematic analysis in psychology. Qualitative Research in Psychology, 3, 77-101.

Brown, K. W., \& Ryan, R. M. (2003). The benefits of being present: Mindfulness and its role in 
psychological well-being. Journal of Personality and Social Psychology, 84, 822-848.

Christopher, J.C.,\& Maris, J.A. (2010) Integrating mindfulness as self-care into counseling and psychotherapy training. Counseling and Psychotherapy Research, 10(2), 114-125.

Cigolla, F., \& Brown, D. (2011). A way of being: Bringing mindfulness into individual therapy. Psychotherapy Research, 21, 709-721.

Coffey, K.A., Hartman, M., \& Fredrickson, B.L. (2010) Deconstructing mindfulness and constructing mental health: understanding mindfulness and its mechanisms of action. Mindfulness, 1, 235-253.

Crane, R., \& Elias, D. (2006). Being with what is: Mindfulness practice for counselors and psychotherapists. Therapy Today, 17, 31-35.

Eifert, G.H., \& Forsyth, J.P. (2005).Acceptance and Commitment Therapy for Anxiety Disorders. Oakland, CA: New Harbinger

Fromm, E. (2002). The Art of Being. NY: Continuum.

Fulton, P.R. (2005). Mindfulness as clinical training. In C.K. Germer, R.D. Siegel, \& P.R. Fulton (Eds.), Mindfulness and psychotherapy (pp. 55-72). New York, NY: Guilford Press.

Gehart, D. B., \& McCollum, E. E. (2008) Inviting therapeutic presence: A mindfulness-based approach. In S. Hick \& T. Bien (Eds.), Mindfulness and the therapeutic relationship (pp. 176-194). New York: Guilford Press.

Germer, C.K. (2005) Teaching Mindfulness in Therapy. In Germer, C.K., Siegel, R.D., Fulton, P.R. (Eds.) Mindfulness and Psychotherapy (pp. 113-129). New York, NY: Guilford Press.

Grepmair, L., Mitterlehner, F., Loew, T., Bachler, E., Rother, W., \& Nickel, M. (2007). Promoting mindfulness in psychotherapists in training influences the treatment results of 
their patients: A randomized, double-blind, controlled study. Psychotherapy and Psychosomatics, 76, 332-338.

Hanh, T. N. (2006). Transformation and Healing. Berkley, CA: Parallax.

Hayes, S.C., Strosahl, K., \& Wilson, K.G. (1999) Acceptance and Commitment Therapy. New York: Guilford Press.

Hick, S. (2008).Cultivating therapeutic relationships: The role of mindfulness. In S. Hick \& T. Bien (Eds.), Mindfulness and the therapeutic relationship (pp. 3-18). New York: Guilford.

Kabat-Zinn, J. (1982). An outpatient program in behavioral medicine for chronic pain patients based on the practice of mindfulness meditation: Theoretical considerations and $\mathrm{p}$ reliminary results. General Hospital Psychiatry, 4, 33-42.

Kabat-Zinn, J. (1994). Wherever you go there you are: Mindfulness meditation in everyday life. New York: Hyperion.

Kabat-Zinn, J. (1990). Full catastrophe living: Using the wisdom of your body and mind to face stress, pain, and illness. New York: Delacorte.

Lambert, M. J. (1992). Implications of outcome research for psychotherapy intergration. In J.C. Norcross and M.R. Goldstein (Eds.), Handbook of psychotherapy integration. New York: Basic Books.

Lesh, T.V. (1970) Zen Meditation and the development of empathy in counselors. Journal of Humanistic Psychology, 10, 39-74.

Linehan, M.M. (1993) Cognitive-behavioral treatment for borderline personality disorder. New York: Guilford Press.

Malpass, A. Havi, C., Ridd, M., Shaw, A., Kessler, D., Sharp, D., Bowden, M., \& Wallond, J. 
(2012). Transforming the Perceptual Situation: a Meta-ethnography of Qualitative Work Reporting Patients’ Experiences of Mindfulness-Based Approaches. Mindfulness, 3, 6075.

McCollum, E. E., \& Gehart, D. R. (2010) Using mindfulness meditation to teach beginning therapists therapeutic presence: A qualitative study. Journal of Marriage and Family Therapy, 36(3), 347-360.

Morgan, W. \& Morgan, S. (2005). Cultivation attention and empathy. In C. Germer, R. Siegel, \& P. Fulton (Eds.), Mindfulness and Psychotherapy. (pp. 73-90).. New York: Guilford Press.

Ostafin, B.D., Chawla, N., Bowen, S., Dillworth, T.M., Witkiewitz, K., \&Marlatt, G.A. (2006). Intensive Mindfulness Training and the Reduction of Psychological Distress: A Preliminary Study. Cognitive and Behavioral Practice, 13, 191-197.

Rogers, C. (1961). On Becoming a Person: A Therapist's View of Psychotherapy. London: Constable.

Ryan, A., Safran, J.D., Doran, J.M., \& Muran, J.C., (2012) Therapist mindfulness, alliance and treatment outcome. Psychotherapy Research, 22, 289-297.

Schure, M.B., Christopher, J., \& Christopher, S. (2008). Mind-Body Medicine and the Art of Self-Care: Teaching Mindfulness to Counseling Students Through Yoga, Meditation, and Qigong.Journal of Counseling and Development, 86(1), 47-56.

Shapiro, S., \& Izett, C.D. (2008). Meditation: A universal tool for cultivating empathy. In S. Hick \& T. Bien (Eds.), Mindfulness and the therapeutic relationship (pp. 161-175). New York: Guilford Press.

Surrey, J.L. (2005). Relational psychotherapy, relational mindfulness. In C.K. Germer, R.D. 
Siegel, \& P.R. Fulton (Eds.), Mindfulness and psychotherapy (pp. 91-112). New York, NY: Guilford Press.

Wilson, K. (2008) Mindfulness for Two: An Acceptance and Commitment Therapy Approach to Mindfulness and Psychotherapy. Oakland, CA: New Harbinger. 
Table 1

Treatment Information for Client/Therapist Dyad Participants

\begin{tabular}{|c|c|c|c|}
\hline & $\begin{array}{l}\text { Length of Treatment } \\
\text { (at time of interview) }\end{array}$ & $\begin{array}{l}\text { Client presenting } \\
\text { problem }\end{array}$ & $\begin{array}{l}\text { Mindfulness } \\
\text { Exercises used }\end{array}$ \\
\hline Dyad 1 & 13 sessions & Social Anxiety/Phobia & $\begin{array}{l}\text { Breathing meditation, } \\
\text { Body-Scan, Loving- } \\
\text { Kindness Meditation }\end{array}$ \\
\hline Dyad 2 & 6 sessions & $\begin{array}{l}\text { Depressive Symptoms } \\
\text { due to a general } \\
\text { medical condition/ } \\
\text { Pain Management }\end{array}$ & $\begin{array}{l}\text { Breathing Meditation, } \\
\text { Body-Scan }\end{array}$ \\
\hline Dyad 3 & 10 sessions & $\begin{array}{l}\text { General Anxiety, } \\
\text { Relational Problems }\end{array}$ & $\begin{array}{l}\text { Breathing Mediation, } \\
\text { Body-Scan }\end{array}$ \\
\hline Dyad 4 & $30+$ sessions & $\begin{array}{l}\text { Depression/Anxiety } \\
\text { symptoms, Family of } \\
\text { Origin issues }\end{array}$ & $\begin{array}{l}\text { Breathing Meditation, } \\
\text { Body Scan, Imagery } \\
\text { Meditation }\end{array}$ \\
\hline Dyad 5 & 20 sessions & $\begin{array}{l}\text { Stress management, } \\
\text { Parenting issues }\end{array}$ & $\begin{array}{l}\text { Thoughts/Emotion } \\
\text { tracking exercise }\end{array}$ \\
\hline
\end{tabular}

